\title{
ON THE CRITICAL LINE OF CONVEX CO-COMPACT HYPERBOLIC SURFACES
}

\author{
DMITRY JAKOBSON AND FRÉDÉRIC NAUD
}

\begin{abstract}
Let $\Gamma$ be a convex co-compact Fuchsian group. We formulate a conjecture on the critical line i.e. what is the largest half-plane with finitely many resonances for the Laplace operator on the infinite area hyperbolic surface $X=\Gamma \backslash \mathbb{H}^{2}$. An upper bound depending on the dimension $\delta$ of the limit set is proved which is in favor of the conjecture for small values of $\delta$ and in the case when $\delta>\frac{1}{2}$ and $\Gamma$ is a subgroup of an arithmetic group. New omega lower bounds for the error term in the hyperbolic lattice point counting problem are derived.
\end{abstract}

\section{INTRODUCTION}

Resonances are the natural replacement data for the missing eigenvalues of the Laplacian when dealing with non compact Riemannian manifolds. In this paper we focus on hyperbolic surfaces. Let $\mathbb{H}^{2}$ be the hyperbolic plane endowed with its standard metric of constant gaussian curvature -1 . Let $\Gamma$ be a geometrically finite Fuchsian group of isometries acting on $\mathbb{H}^{2}$. This means that $\Gamma$ admits a finite sided polygonal fundamental domain in $\mathbb{H}^{2}$. We will require that $\Gamma$ has no elliptic elements different from the identity and that the quotient $\Gamma \backslash \mathbb{H}^{2}$ is of infinite hyperbolic area. We assume in addition in this paper that $\Gamma$ has no parabolic elements (no cusps). Under these assumptions, the quotient space $X=\Gamma \backslash \mathbb{H}^{2}$ is a Riemann surface (called convex co-compact) whose geometry can be described as follows. The surface $X$ can be decomposed into a compact surface $N$ with geodesic boundary, called the Nielsen region, on which $f(f \geq 1)$ infinite area ends $F_{i}$ are glued : the funnels. A funnel $F_{i}$ is a half cylinder

$$
F_{i}=\left(\mathbb{R} / l_{i} \mathbb{Z}\right)_{\theta} \times\left(\mathbb{R}^{+}\right)_{t},
$$

where $l_{i}>0$, with the warped metric

$$
d s^{2}=d t^{2}+\cosh ^{2}(t) d \theta^{2} .
$$

An important numerical datum related to the group $\Gamma$ is the Hausdorff dimension of its limit set $\delta(\Gamma)$. The limit set is commonly defined by

$$
\Lambda(\Gamma):=\overline{\Gamma . z} \cap \partial \mathbb{H}^{2}
$$

where $z \in \mathbb{H}^{2}$ is a given point and $\Gamma . z$ is the orbit of that point under the action of $\Gamma$ which by discreteness accumulates on the boundary $\partial \mathbb{H}^{2}$. The limit set $\Lambda$ does not depend on the choice of $z$ and its Hausdorff 
dimension $\delta(\Gamma)$ is the critical exponent of Poincaré series [23] (here $d$ denotes hyperbolic distance)

$$
P_{\Gamma}(s):=\sum_{\gamma \in \Gamma} e^{-s \mathrm{~d}\left(\gamma z, z^{\prime}\right)} .
$$

Let $\Delta_{X}$ be the hyperbolic Laplacian on $X$. Its spectrum on $L^{2}(X)$ has been described completely by Lax and Phillips in [18]. The half line $[1 / 4,+\infty)$ is the continuous spectrum and it contains no embedded eigenvalues. The rest of the spectrum (point spectrum) is empty if $\delta \leq \frac{1}{2}$, finite and starting at $\delta(1-\delta)$ if $\delta>\frac{1}{2}$. The fact that the bottom of the spectrum is related to the dimension $\delta$ was first discovered by Patterson [23] for convex co-compact groups.

By the preceding description of the spectrum, the resolvent

$$
R_{\Gamma}(s)=\left(\Delta_{X}-s(1-s)\right)^{-1}: L^{2}(X) \rightarrow L^{2}(X),
$$

is therefore well defined and analytic on the half-plane $\left\{\operatorname{Re}(s)>\frac{1}{2}\right\}$ except at a possible finite set of poles corresponding to the finite point spectrum. Resonances are then defined as poles of the meromorphic continuation of

$$
R_{\Gamma}(s): C_{0}^{\infty}(X) \rightarrow C^{\infty}(X)
$$

to the whole complex plane. The set of poles is denoted by $\mathcal{R}_{X}$. This continuation is usually performed via the analytic Fredholm theorem after the construction of an adequate parametrix. The first result of this kind in the more general setting of asymptotically hyperbolic manifolds is due to Mazzeo and Melrose [20]. A more precise parametrix for surfaces was constructed by Guillopé and Zworski in [14, 13]. It should be mentioned at this point that in the infinite area case, resonances are spread all over the half plane $\left\{\operatorname{Re}(s)<\frac{1}{2}\right\}$, in sharp contrast with the finite area non compact case where resonances are known to be confined in a strip. In this note, we adress the problem of finding resonances with the largest real part (which roughly speaking measures the decay rate of solutions of the automorphic wave equation). By Lax-Phillips theory, we know that when $\delta>\frac{1}{2}$, all resonances (except the finite set of genuine $L^{2}$-eigenvalues) are in the half plane $\left\{\operatorname{Re}(s)<\frac{1}{2}\right\}$. On the other hand, if $\delta \leq \frac{1}{2}$ and $\Gamma$ is a non elementary group (which means that $\Gamma$ is not generated by a single hyperbolic elements and its inverse), a result of the second author [21] shows that there exists $\epsilon>0$ such that $\mathcal{R}_{X} \cap\{\operatorname{Re}(s) \geq \delta-\epsilon\}=\{\delta\}$. The constant $\epsilon$ obtained as a byproduct of a certain Dolgopyat estimate is not effective.

This picture leads to the natural (and likely difficult) question: what is the actual size of the "essential spectral gap" or locate the critical 
line for the resonances in the half plane $\{\operatorname{Re}(s) \leq \delta\}$. In other words, can one compute

$$
G(\Gamma):=\inf \left\{\sigma \in \mathbb{R}:\{\operatorname{Re}(s) \geq \sigma\} \cap \mathcal{R}_{X} \text { is finite }\right\} .
$$

Clearly by the above discussion, we do have $G(\Gamma) \leq \frac{1}{2}$ if $\delta>\frac{1}{2}$ and $G(\Gamma)<\delta$ if $\delta \leq \frac{1}{2}$ and $\Gamma$ is non-elementary. On the other hand, it follows from the existence of one closed primitive geodesic for non trivial $\Gamma$ that $G(\Gamma)>-\infty$, see [11]. If $\Gamma$ is elementary resonances can be explicitly computed, see [7], and we have obviously $G(\Gamma)=0=\delta$. We propose the following conjecture, see $\S 2$ for some heuristic justifications.

Conjecture 1.1. Let $\Gamma$ be a convex co-compact group as above, then we have

$$
G(\Gamma)=\frac{\delta}{2}
$$

This conjecture is consistent with the fact that in the finite volume case $(\delta=1)$, a result of Selberg [26] shows that there exist infinitely many resonances in the half-plane

$$
\left\{\operatorname{Re}(s)<\frac{1}{2}\right\}
$$

whose real parts are as close to $\frac{1}{2}$ as we want, hence $G(\Gamma)=\frac{1}{2}$ for co-finite groups. However, his strategy does not work in the infinite volume case, mostly because resonances dot not lie in a strip. In this paper, we prove the following.

Theorem 1.2. Let $\Gamma$ be a convex co-compact Fuchsian group, then we always have $G(\Gamma) \geq \frac{\delta(1-2 \delta)}{2}$. If in addition $\frac{1}{2}<\delta$ and $\Gamma$ is a convex co-compact subgroup of an arithmetic group, then $G(\Gamma) \geq \frac{\delta}{2}-\frac{1}{4}$.

We will give more details about what arithmetic groups are (or rather what we need about them) in $\S 3.2$. We notice that as $\delta \rightarrow 0$, we have $G(\Gamma) \geq \frac{\delta}{2}+O\left(\delta^{2}\right)$ so our lower bound is, at least infinitesimally, in favor of the conjecture. The above estimate clearly beats previous results on strips with infinitely many resonances obtained for arbitrary convex co-compact hyperbolic manifolds in [10].

It follows from a recent result of Lewis Bowen [5] (see also [8, 3] for the $\mathrm{SL}_{2}(\mathbb{Z})$ case) that in every co-finite or co-compact arithmetic Fuchsian group, one can find convex co-compact subgroups with dimension arbitrarily close to 1 . So there are plenty of examples of convex cocompact surfaces with $G(\Gamma) \geq 1 / 4-\epsilon, \epsilon$ being arbitrarily small.

One of the motivations for this paper is the hyperbolic lattice point counting problem. Indeed, a famous result of Lax-Phillips [17] shows that for $\delta>\frac{1}{2}$, for all $z, z^{\prime} \in \mathbb{H}^{2}$, there exists a finite asymptotic expansion of the counting function ("d" denotes hyperbolic distance)

$$
N\left(T ; z, z^{\prime}\right):=\#\left\{\gamma \in \Gamma: \mathrm{d}\left(z, \gamma z^{\prime}\right) \leq T\right\},
$$


which is as $T \rightarrow+\infty$

$$
N\left(T ; z, z^{\prime}\right)=\sum_{j} C_{j}\left(z, z^{\prime}\right) e^{\delta_{j} T}+O\left(T^{5 / 6} e^{(\delta+1) T / 3}\right),
$$

where $j$ runs over a finite set and each $\delta_{j} \in\left(\frac{1}{2}, \delta\right]$ is related to a small eigenvalue of $\Delta_{X}$ through the formula $\delta_{j}\left(1-\delta_{j}\right)=\Gamma_{j}, \delta_{0}=\delta$. It is conjectured in the finite volume case that the optimal error term is $O\left(e^{(1 / 2+\epsilon) T}\right)$. In the infinite volume case, in view of our conjecture, the optimal error term should be $O\left(e^{(\delta / 2+\epsilon) T}\right)$ and one would a priori need an expansion involving also resonances in addition to $L^{2}$ point spectrum. If $\delta \leq \frac{1}{2}$, then Lax-Phillips theory no longer applies. It follows from the work of Lalley [16] and the second author [21], see also [4], that one can find $\epsilon_{0}>0$ such that for large $T$,

$$
N\left(T ; z, z^{\prime}\right)=C\left(z, z^{\prime}\right) e^{\delta T}+O\left(e^{\left(\delta-\epsilon_{0}\right) T}\right) .
$$

The relationship between the error term in the counting problem and the essential spectral gap $G(\Gamma)$ is the following result which might be viewed as some kind of "abstract non-sense".

Theorem 1.3. Let $\Gamma$ be a convex co-compact Fuchsian Group and assume that $G(\Gamma)>0$. There exists a full measure subset $\mathcal{G} \subset \mathbb{H}^{2} \times \mathbb{H}^{2}$ such that for all $\left(z, z^{\prime}\right) \in \mathcal{G}$ and all finite expansion of the form

$$
\sum_{j} Q_{j}\left(T ; z, z^{\prime}\right) e^{\delta_{j} T}
$$

where $\delta_{j} \in \mathbb{C}$ and $Q_{j}\left(T ; z, z^{\prime}\right)$ is a polynomial $\in \mathbb{C}[T]$, we have for all $\epsilon>0$ and as $T \rightarrow+\infty$,

$$
\left|N\left(T ; z, z^{\prime}\right)-\sum_{j} Q_{j}\left(T ; z, z^{\prime}\right) e^{\delta_{j} T}\right|=\Omega\left(e^{(G(\Gamma)-\epsilon) T}\right) .
$$

The notation $\Omega(\bullet)$ means, as usual in number theory, being not a $O(\bullet)$. In view of our above lower bounds, we have in the case when $\Gamma$ is arithmetic with $\delta>\frac{1}{2}$, an omega lower bound for the remainder of size $\Omega\left(e^{(\delta / 2-1 / 4-\epsilon) T}\right)$, hence the errror term in the expansion cannot be bounded.

The plan of the paper is as follows. In section $\S 2$, we motivate conjecture 1.1 by some heuristics based on thermodynamical formalism that might lead to a future proof. We then explain the application to the lattice point counting problem. Although it is a fairly direct consequence of the previous result, some details have to be provided, in particular we explain in terms of the polar structure of the resolvent where the generic set of $\left(z, z^{\prime}\right)$ for which the lower bound holds comes from. In section $\S 3$ we prove Theorem 1.2. We first prove an approximate Trace formula to take advantage of the known upper bounds on Selberg's zeta function. The result then follows from the prime geodesic theorem and a mean square argument "à la Selberg". Arithmeticity is 
used when $\delta>\frac{1}{2}$ to improve lower bounds, using exponentially growing multiplicities in the length spectrum.

Finally, we mention that using the trace formula from [9] or the Patterson-Perry result on the divisor of the Selberg zeta function [24], our result should extend to higher dimensional convex co-compact hyperbolic manifolds of Schottky type (the fractal Weyl upper bound from Guillopé-Lin-Zworski [12], see estimate (2) below, is critical in our proof). If the fractal Weyl upper bound (2) holds for surfaces with cusps, then our result should extend straightforwardly to that case with the same lower bounds.

Acknowledgement. We both thank the two referees for their serious reading and comments that definitely helped to improve the paper. We thank Peter Sarnak for pointing out reference [5]. Both Authors thank for their hospitality the Banff center and McGill university where part of this work has been done. D.J. is supported by NSERC, FQRNT and Dawson fellowship. F.N. is supported by ANR METHCHAOS.

\section{Heuristics on the CONJECTURE AND motivations}

2.1. Transfer operators and zeta function. In the sequel we assume that $\Gamma$ is a convex co-compact non elementary Fuchsian group. Recall that Selberg's zeta function $Z_{\Gamma}(s)$ is defined for $\operatorname{Re}(s)$ large by the infinite product

$$
Z_{\Gamma}(s):=\prod_{n \in \mathbb{N}} \prod_{\gamma \in \mathcal{P}}\left(1-e^{-(s+n) l(\gamma)}\right),
$$

where $\mathcal{P}$ is the set of prime closed geodesics on $X=\Gamma \backslash \mathbb{H}^{2}$ and if $\gamma \in \mathcal{P}$, $l(\gamma)$ is the length of the prime geodesic $\gamma$. By a rough upper bound on the number of closed geodesics, convergence of the above product actually occurs for all $\operatorname{Re}(s)>\delta$. Since $\Gamma$ is a convex co-compact group, it is known that the above product extends analytically to an entire function on the whole complex plane, see [2]. In addition, the work of Patterson-Perry [24] tells that the non-trivial zeros are the resonance set $\mathcal{R}_{X}$. The largest zero (in term of its real part) of $Z_{\Gamma}(s)$ is at $s=\delta$ and is known to be simple. There are no other zeros on the line $\{\operatorname{Re}(s)=\delta\}$. To investigate zeros of Selberg zeta function one can try to use the fact that it is a Fredholm determinant of a holomorphic family of trace class operators called "Ruelle transfer operators" $L_{s}$ so that

$$
Z_{\Gamma}(s)=\operatorname{det}\left(I-L_{s}\right) .
$$

Let us be more specific. Let $\mathcal{D}_{1}, \ldots, \mathcal{D}_{p} ; \mathcal{D}_{p+1}, \ldots \mathcal{D}_{2 p}, p \geq 2$ be $2 p$ closed, disjoints, euclidean discs in $\mathbb{C}$ which we take to be orthogonal to $\partial \mathbb{H}^{2}$ (we use for example the upper half plane model). Let $h_{1}, \ldots, h_{p} \in$ 
$\mathrm{PSL}_{2}(\mathbb{R})$ be such that for all $i=1, \ldots, p$

$$
h_{i}\left(\mathcal{D}_{i}\right)=\overline{\widehat{\mathbb{C}} \backslash \mathcal{D}_{p+i}} \text {. }
$$

We recall that the group $\mathrm{PSL}_{2}(\mathbb{R})$ acts on the Poincaré half-plane (and the Riemann sphere $\widehat{\mathbb{C}}$ ) by Möbius transformations

$$
h(z)=\frac{a z+b}{c z+d} ; a d-b c=1 .
$$

The (free) group $\Gamma$ generated by $h_{1}, \ldots, h_{p} ; h_{1}^{-1}, \ldots, h_{p}^{-1}$ is convex cocompact (the converse is also true, every convex co-compact subgroup of $\mathrm{PSL}_{2}(\mathbb{R})$ can be realized as a Schottky group, see for example [1] chapter 15). For convenience we set $h_{p+i}=h_{i}^{-1}$ for all $i=1, \ldots, p$. For all $i$, set $I_{i}:=\partial \mathbb{H}^{2} \cap \mathcal{D}_{i}$. The Bowen-Series map is an (eventually) expanding map defined on $I:=\cup_{i=1}^{2 p} I_{i}$ by $B(x)=h_{i}(x)$ if $x \in I_{i}$ as a map

$$
B: I \rightarrow \mathbb{R} \cup\{\infty\} .
$$

The forward invariant subset of $\partial \mathbb{H}^{2}$ defined by $\cap_{n \in \mathbb{N}} B^{-n}(I)$ is the limit set $\Lambda(\Gamma)$. Set

$$
\mathcal{U}=\bigcup_{i=1}^{2 p} \mathcal{D}_{i},
$$

then the Bowen series map $B$ clearly also makes sense on $\mathcal{U}$ as a map

$$
B: \mathcal{U} \rightarrow \widehat{\mathbb{C}} \text {. }
$$

We define the function space $\mathcal{A}(\mathcal{U})$ to be

$$
\mathcal{A}(\mathcal{U}):=\left\{f \in L^{2}(\mathcal{U}): f \text { is holomorphic on } \mathcal{U}\right\} .
$$

The Ruelle transfer operator $L_{s}: \mathcal{A}(\mathcal{U}) \rightarrow \mathcal{A}(\mathcal{U})$ is defined (see [12]) for $z \in \mathcal{D}_{i}$ by

$$
L_{s}(f)(z):=\sum_{j \in\{1, \ldots, 2 p\} \backslash\{i\}}\left(h_{j}^{\prime}(z)\right)^{s} f\left(h_{j}(z)\right)=\sum_{y: B(y)=z}\left|B^{\prime}(y)\right|^{-s} f(y) .
$$

Note that for all $j \neq i$, each $h_{j}: \mathcal{D}_{i} \rightarrow \mathcal{D}_{p+j}$ (indexes have to be understood $\bmod 2 p$ ) is a holomorphic contraction in the sense that $h_{j}\left(\mathcal{D}_{i}\right) \subset \operatorname{Int}\left(\mathcal{D}_{p+j}\right)$. It has been known since Ruelle [25] that these operators are compact, trace class. The exact computation of the trace then shows that

$$
Z_{\Gamma}(s)=\operatorname{det}\left(I-L_{s}\right) .
$$

We warn the reader that we enter below the (very) non-rigourous part of this "heuristics" subsection whose purpose is only to suggest that our conjecture is "plausible". Writing $s=\sigma+i t$, we can observe that $(x \in I)$

$$
\begin{gathered}
\left|L_{s}^{N}(f)(x)\right|^{2}= \\
\sum_{y, w}\left|\left(B^{N}\right)^{\prime}(y)\right|^{-\sigma}\left|\left(B^{N}\right)^{\prime}(w)\right|^{-\sigma} e^{-i t\left(\log \left|\left(B^{N}\right)^{\prime}(y)\right|-\log \mid\left(B^{N}\right)^{\prime}(w)\right) \mid} f(y) \bar{f}(w),
\end{gathered}
$$


where the double sum runs over all $y, w$ such that $B^{N} y=x, B^{N} w=x$. If one believes that, in the regime $|t|>>1$ and $N$ of size $C \log |t|^{1}$, the off-diagonal terms in the above sum will cancel enough so that the main contribution comes from the diagonal terms $w=y$, we have

$$
\left|L_{s}^{N}(f)(x)\right|^{2}=O_{f}\left(\sum_{B^{N} y=x} e^{-2 \sigma \log \left|\left(B^{N}\right)^{\prime}(y)\right|}\right)
$$

It is known that this above sum is related to a quantity called "topological pressure" i.e. as $N \rightarrow \infty$,

$$
\lim _{N \rightarrow+\infty}\left(\sum_{B^{N} y=x} e^{-2 \sigma \log \left|\left(B^{N}\right)^{\prime}(y)\right|}\right)^{1 / N}=e^{P(-2 \sigma)} .
$$

The topological pressure is a thermodynamical quantity related to the Bowen map on the boundary by the following variational formula, $(\sigma \in$ $\mathbb{R})$

$$
P(-\sigma)=\sup _{\mu}\left(h_{\mu}(B)-\sigma \int_{\Lambda(\Gamma)} \log \left|B^{\prime}\right| d \mu\right),
$$

where the sup is understood over all $B$-invariant probability measures supported on the limit set $\Lambda$ and $h_{\mu}$ is the measure theoretic entropy. The map $\sigma \mapsto P(-\sigma)$ is strictly decreasing, convex, and Bowen's formula (see [6]) says that it has a unique zero at $\sigma=\delta$. One is then tempted to believe that on a suitable function space, the operators $L_{s}$ will be eventually contracting for $\operatorname{Re}(s)>\delta / 2$ and $|\operatorname{Im}(s)|$ large. Therefore it seems that the determinant

$$
Z_{\Gamma}(s)=\operatorname{det}\left(I-L_{s}\right)
$$

is non vanishing for $\operatorname{Re}(s)>\delta / 2$ and $\operatorname{Im}(s)$ large, i.e. $G(\Gamma) \leq \delta / 2$.

On the other hand, it follows rigourously from [22] that for all $\operatorname{Re}(s)=\sigma$ fixed, for all $\epsilon>0$, one can find a sequence $\left(t_{k}\right)$ with $\left|t_{k}\right| \rightarrow+\infty$ as $k \rightarrow+\infty$ such that the spectral radius $\rho\left(L_{\sigma+i t_{k}}\right)$ satisfies the lower bound

$$
\rho\left(L_{\sigma+i t_{k}}\right) \geq e^{\frac{3}{2} P(-2 \sigma)},
$$

which shows that for $\operatorname{Re}(s)<\delta / 2$, the operators $L_{s}$ are no longer (eventually) contracting as $|\operatorname{Im}(s)|$ goes to infinity, which is in favor of $G(\Gamma) \geq \delta / 2$. Of course having $\rho\left(L_{\sigma+i t_{k}}\right) \geq 1$ is not enough to produce a zero of the Selberg zeta function (we actually need 1 to be an eigenvalue) and clearly some deeper knowledge on the spectral theory of these transfer operator has to be gained to reach the conjecture.

\footnotetext{
${ }^{1}$ One of the difficulties is to move from iterates of order $O(\log |t|)$ to arbitrary $N$. This is achieved in the implementation of Dolgopyat method [21] but there very few cancellations are used, resulting in a crude, non-effective bound. We also elude here the question of which function space is relevant for these computations.
} 
2.2. The error term in the hyperbolic lattice counting prob-

lem. In this subsection, we prove the omega lower bound for the error term in Lax-Phillips lattice counting asymptotic. Let us assume that we have for fixed $z, z^{\prime}$ in $\mathbb{H}^{2}$ with $z \neq z^{\prime}$ in $\Gamma \backslash \mathbb{H}^{2}$ and $0<\rho<\delta$,

$$
N\left(T ; z, z^{\prime}\right)=\sum_{j} Q_{j}\left(T ; z, z^{\prime}\right) e^{\delta_{j} T}+O\left(e^{\rho T}\right),
$$

where $j$ runs over a finite set, $\delta_{j} \in \mathbb{C}$ and

$$
Q_{j}\left(T, z, z^{\prime}\right)=\sum_{k=0}^{N(j)} a_{k, j}\left(z, z^{\prime}\right) T^{k}
$$

The usual Poincaré series defined for $\operatorname{Re}(s)>\delta$ by

$$
P_{\Gamma}\left(s ; z, z^{\prime}\right):=\sum_{\gamma \in \Gamma} e^{-s \mathrm{~d}\left(z, \gamma z^{\prime}\right)},
$$

are related to the counting function $N\left(T ; z, z^{\prime}\right)$ via summation by parts through the formula

$$
P_{\Gamma}\left(s ; z, z^{\prime}\right)=s \int_{0}^{+\infty} e^{-s t} N\left(t ; z, z^{\prime}\right) d t .
$$

Inserting the asymptotic in this formula we get for $\operatorname{Re}(s)>\delta$,

$$
P_{\Gamma}\left(s ; z, z^{\prime}\right)=\sum_{j} \sum_{k=0}^{N(j)} a_{k, j}\left(z, z^{\prime}\right) \frac{s k !}{\left(s-\delta_{j}\right)^{k+1}}+F\left(s ; z, z^{\prime}\right),
$$

where $s \mapsto F\left(s ; z, z^{\prime}\right)$ has a holomorphic extension to the half plane

$$
\{\operatorname{Re}(s)>\rho\} .
$$

Poincaré series are in turn related to the resolvent kernel $R_{\Gamma}\left(s ; z, z^{\prime}\right)$ on $\Gamma \backslash \mathbb{H}^{2}$ by the Gauss hypergeometric function averaged over the group $\Gamma$. More precisely, for $\operatorname{Re}(s)>\delta$ and $z \neq z^{\prime}$ in $\Gamma \backslash \mathbb{H}^{2}$, we have

$$
R_{\Gamma}\left(s ; z, z^{\prime}\right)=\sum_{\gamma \in \Gamma} G_{s}\left(\sigma\left(z, \gamma z^{\prime}\right)\right),
$$

with $\sigma\left(z, z^{\prime}\right)=\cosh ^{2}\left(\mathrm{~d}\left(z, z^{\prime}\right) / 2\right)$ and for $\sigma>1$,

$$
G_{s}(\sigma)=\frac{1}{4 \pi} \sum_{k=0}^{\infty} \frac{(\Gamma(s+k))^{2}}{k ! \Gamma(2 s+k)} \sigma^{-s-k} .
$$

From that fact one infers that there exists a holomorphic function on the half plane

$$
\{\operatorname{Re}(s)>\delta-1\}
$$

denoted by $H\left(s ; z, z^{\prime}\right)$ such that

$$
R_{\Gamma}\left(s ; z, z^{\prime}\right)=\frac{4^{s-1}}{\pi} \frac{(\Gamma(s))^{2}}{\Gamma(2 s)} P_{\Gamma}\left(s ; z, z^{\prime}\right)+H\left(s ; z, z^{\prime}\right)
$$


see [1], proposition 14.17 for computational details. We have therefore shown that for $\left(z, z^{\prime}\right)$ away from the diagonal in $\Gamma \backslash \mathbb{H}^{2} \times \Gamma \backslash \mathbb{H}^{2}$, the map

$$
s \mapsto R_{\Gamma}\left(s ; z, z^{\prime}\right)
$$

extends meromorphically to $\{\operatorname{Re}(s)>\rho\}$ with only finitely many poles.

On the other hand, we know from the parametrix of Guillopé-Zworski that (again away from the diagonal) $s \mapsto R_{\Gamma}\left(s ; z, z^{\prime}\right)$ has a meromorphic extension to $\mathbb{C}$. The structure of this kernel at a resonance is as follows, see [1] proposition 8.1. Assume that $\zeta \in \mathbb{C}$ is a pole, then there exist $p_{\zeta} \geq 1$ and a finite family $A_{j}\left(\zeta ; z, z^{\prime}\right)$ of smooth, finite rank kernels such that

$$
R_{\Gamma}\left(s ; z, z^{\prime}\right)=\sum_{j=1}^{p} \frac{A_{j}\left(\zeta ; z, z^{\prime}\right)}{(s(1-s)-\zeta(1-\zeta))^{j}}+E_{\zeta}\left(s ; z, z^{\prime}\right),
$$

where $s \mapsto E_{\zeta}\left(s ; z, z^{\prime}\right)$ is holomorphic on a neighborhood of $\zeta$. The residue $A_{1}\left(\zeta ; z, z^{\prime}\right)$ is always non-trivial as a function of $\left(z, z^{\prime}\right)$ and can be described more precisely as

$$
A_{1}\left(\zeta ; z, z^{\prime}\right)=\sum_{k=1}^{m_{\zeta}} \phi_{k, \zeta}(z) \phi_{k, \zeta}\left(z^{\prime}\right)
$$

where each $\phi_{k, \zeta} \in C^{\infty}(X), X=\Gamma \backslash \mathbb{H}^{2}$, is a non-trivial solution of the elliptic equation

$$
\left(\Delta_{X}-s(1-s)\right)^{p} \phi=0 .
$$

The set of functions $\left\{\phi_{k, \zeta}\right\}_{k=1}^{m_{\zeta}}$ is a basis for the range of $A_{1}(\zeta)$ acting as an operator in certain weighted $L^{2}$ spaces, we refer the reader to [1], prop. 8.1 for further details. The coefficients of the elliptic operator $\left(\Delta_{X}-s(1-s)\right)^{p}$ being obviously real-analytic, by a well known theorem, each $\phi_{k, \zeta}$ is a (possibly $\mathbb{C}$-valued) non-trivial real-analytic function on $X$. Therefore the function

$$
\left(z, z^{\prime}\right) \mapsto A_{1}\left(\zeta ; z, z^{\prime}\right)
$$

is real-analytic (and non trivial as a function of $\left(z, z^{\prime}\right)$ ) therefore its zero set $z(\zeta) \subset X \times X$ has zero Lebesgue measure. Now fix an $\epsilon_{0}>0$ and consider the set

$$
\mathcal{N}:=\bigcup_{\operatorname{Re}(\zeta) \geq G(\Gamma)-\epsilon_{0}} z(\zeta),
$$

where the union is understood over the (infinite) countable set of all resonances in the half-plane $\left\{\operatorname{Re}(\zeta) \geq G(\Gamma)-\epsilon_{0}\right\}$. Clearly the Lebesgue measure of $\mathcal{N} \subset X \times X$ is zero. Consider now

$$
\mathcal{G}:=X \times X \backslash(\mathcal{N} \cup \Delta),
$$

where $\Delta$ stands for the diagonal in $X \times X$. The set $\mathcal{G}$ is of full Lebesgue measure and for each $\left(z, z^{\prime}\right) \in \mathcal{G}$ the kernel resolvent $s \mapsto R_{\Gamma}\left(s ; z, z^{\prime}\right)$ has infinitely many poles in $\{\operatorname{Re}(s) \geq G(\Gamma)-\epsilon\}$ for all $0<\epsilon \leq \epsilon_{0}$. We have a contradiction whenever $\rho \leq G(\Gamma)-\epsilon$. 


\section{Proof of the main Result}

We assume throughout this section that $\Gamma$ is a convex co-compact Fuchsian group which is non elementary. This causes no trouble since the elementary case $(\delta=0)$ is well understood in term of resonances [7]. Although we could obtain Theorem 1.2 from the Guillopé-Zworski 0 -trace formula [11], it will prove more efficient for us to work directly with Selberg's zeta function instead.

3.1. An approximate trace formula. Working with Selberg's zeta function plus an estimate due to Guillopé and Zworski allows us to obtain an approximate trace formula. Let $\varphi \in C_{0}^{\infty}(\mathbb{R})$ be a smooth compactly supported test function. We define $\psi(s)$ by the formula

$$
\psi(s):=\int_{-\infty}^{+\infty} e^{s u} \varphi(u) d u=\widehat{\varphi}(i s),
$$

where $\widehat{\varphi}$ is the usual Fourier transform of $\varphi$. We have the following claim.

Proposition 3.1. Assume that for a given $\rho<\delta$, the set

$$
\mathcal{F}_{\rho}:=\mathcal{R}_{X} \cap\{\operatorname{Re}(s)>\rho\}
$$

is finite, then for all $\varepsilon>0$ small enough, we have

$$
\begin{aligned}
& \sum_{k \in \mathbb{N}_{0}} \sum_{\gamma \in \mathcal{P}} \frac{l(\gamma)}{1-e^{-k l(\gamma)}} \varphi(k l(\gamma))=\sum_{z \in \mathcal{F}_{\rho}} \psi(z) \\
& +O\left(\int_{-\infty}^{+\infty}(1+|x|)^{\delta}|\psi(\rho+\varepsilon+i x)| d x\right) .
\end{aligned}
$$

The implied constant depends only on $\varepsilon, \rho$ and $\Gamma$.

Proof. First we recall that for $\operatorname{Re}(s)>\delta$, we have the convergent series formula for the logarithmic derivative

$$
\frac{Z_{\Gamma}^{\prime}(s)}{Z_{\Gamma}(s)}=\sum_{(n, \gamma) \in \mathbb{N} \times \mathcal{P}} \frac{l(\gamma) e^{-(s+n) l(\gamma)}}{1-e^{-(s+n) l(\gamma)}}=\sum_{k \in \mathbb{N}_{0}} \sum_{\gamma \in \mathcal{P}} \frac{l(\gamma) e^{-s k l(\gamma)}}{1-e^{-k l(\gamma)}} .
$$

In addition, for all $A>\delta$, one can find a constant $C_{A} \geq 0$ such that for all $\operatorname{Re}(s) \geq A$, we have

$$
\left|\frac{Z_{\Gamma}^{\prime}(s)}{Z_{\Gamma}(s)}\right| \leq C_{A}
$$

Let us consider the contour integral

$$
I_{A}:=\frac{1}{2 i \pi} \int_{A-i \infty}^{A+i \infty} \frac{Z_{\Gamma}^{\prime}(s)}{Z_{\Gamma}(s)} \psi(s) d s,
$$

where $\psi(s)=\widehat{\varphi}(i s)$. Since $\varphi \in C_{0}^{\infty}(\mathbb{R}), \psi(s)$ is rapidly decreasing (Schwartz class) on every vertical line which guarantees convergence of 
the above integral. Using Lebesgue dominated convergence theorem, we can interchange $\sum$ and $\int$ to write

$$
I_{A}=\sum_{k \in \mathbb{N}_{0}} \sum_{\gamma \in \mathcal{P}} \frac{l(\gamma) e^{-A k l(\gamma)}}{1-e^{-k l(\gamma)}} \times \frac{1}{2 \pi} \int_{-\infty}^{+\infty} e^{\widehat{A x} \varphi(x)}(\xi) e^{i \xi k l(\gamma)} d \xi .
$$

Fourier inversion formula yields

$$
I_{A}=\sum_{k \in \mathbb{N}_{0}} \sum_{\gamma \in \mathcal{P}} \frac{l(\gamma)}{1-e^{-k l(\gamma)}} \varphi(k l(\gamma)) .
$$

Assuming that $Z_{\Gamma}(s)$ has only finitely many zeros inside $\{\operatorname{Re}(s)>\rho\}$, the next step is to deform the contour integral $I_{A}$. To perform this contour shift, we obviously need an a priori estimate on the growth of $Z_{\Gamma}^{\prime}(s) / Z_{\Gamma}(s)$. We show the following.

Lemma 3.2. For all $\varepsilon>0$, there exist constants $C>0$ and $R_{0} \geq 0$, such that for all $\operatorname{Re}(s) \geq \rho+\varepsilon$ and $|\operatorname{Im}(s)| \geq R_{0}$, we have

$$
\left|\frac{Z_{\Gamma}^{\prime}(s)}{Z_{\Gamma}(s)}\right| \leq C|\operatorname{Im}(s)|^{\delta} .
$$

Proof. From the scale-analysis of Guillopé-Lin-Zworski [12], it is known that in every half-plane $\{\operatorname{Re}(s) \geq \sigma\}$, one can find $C_{\sigma} \geq 0$ such that

$$
\left|Z_{\Gamma}(s)\right| \leq C_{\sigma} e^{C_{\sigma}|\operatorname{Im}(s)|^{\delta}} .
$$

For all $|t|$ large, the holomorphic function

$$
z \mapsto \frac{Z_{\Gamma}(z+A+i t)}{Z_{\Gamma}(A+i t)}
$$

does not vanish on a neighborhood of the closed disc $\bar{D}(0, A-\rho-\varepsilon / 2)$ so that we can define a complex holomorphic logarithm

$$
f_{t}(z):=\log \left(\frac{Z_{\Gamma}(z+A+i t)}{Z_{\Gamma}(A+i t)}\right)
$$

with $f_{t}(0)=0$ and

$$
\operatorname{Re}\left(f_{t}(z)\right)=\log \left|\frac{Z_{\Gamma}(z+A+i t)}{Z_{\Gamma}(A+i t)}\right| .
$$

We can now apply the Borel-Caratheodory estimate that can be found in Titchmarsh [28].

Lemma 3.3. Assume that $f$ is a holomorphic function on a neighborhood of the closed disc $\bar{D}(0, R)$, and $f(0)=0$. Then for all $r<R$, we have

$$
\max _{|z| \leq r}\left|f^{\prime}(z)\right| \leq \frac{8 R}{(R-r)^{2}} \max _{|z| \leq R}|\operatorname{Re}(f(z))|
$$


Applying the above estimate to $f_{t}$, we eventually obtain for all $s$ in the closed disc $\bar{D}(A+i t, A-\rho-\varepsilon)$, with $|t|$ large,

$$
\left|\frac{Z_{\Gamma}^{\prime}(s)}{Z_{\Gamma}(s)}\right| \leq \frac{16 A}{\varepsilon}\left(C_{\rho}|\operatorname{Im}(s)|^{\delta}+\log C_{\rho}-\log \left|Z_{\Gamma}(A+i t)\right|\right) .
$$

Since $\left|Z_{\Gamma}(s)\right|$ is uniformly bounded from below on $\{\operatorname{Re}(s) \geq A\}$, we have proved the lemma.

We can now conclude the proof of the approximate trace formula. Since $\mathcal{F}_{\rho}$ is finite, for all $\varepsilon>0$ small enough, $Z_{\Gamma}(s)$ does not vanish on the line $\{\operatorname{Re}(s)=\rho+\varepsilon\}$. By the residue theorem we write for $R$ large,

$$
\begin{gathered}
I_{A, R}:=\frac{1}{2 i \pi} \int_{A-i R}^{A+i R} \frac{Z_{\Gamma}^{\prime}(s)}{Z_{\Gamma}(s)} \psi(s) d s \\
=\sum_{\Gamma \in \mathcal{F}_{\rho}} \psi(\Gamma)+\underbrace{\frac{1}{2 i \pi} \int_{\rho+\varepsilon-i R}^{\rho+\varepsilon+i R} \frac{Z_{\Gamma}^{\prime}(s)}{Z_{\Gamma}(s)} \psi(s) d s}_{\widetilde{I}_{\rho, R}}-\underbrace{\frac{1}{2 i \pi} \int_{\substack{\operatorname{Im}(s)= \pm R \\
\rho+\widetilde{\varepsilon} \leq \operatorname{Re}(s) \leq A}} \frac{Z_{\Gamma}^{\prime}(s)}{Z_{\Gamma}(s)} \psi(s) d s .}_{E_{R}}
\end{gathered}
$$

Using the bound (1) plus the rapid decay of $\psi(s)$ on vertical lines, we get that $E_{R} \rightarrow 0$ as $R \rightarrow+\infty$. We therefore have the desired formula by taking the limit as $R \rightarrow+\infty$ and use again the bound (1) in $\widetilde{I}_{\rho, R}$.

3.2. Facts on the arithmetic length spectrum. Given a Fuchsian group $\Gamma$, one defines the lengh spectrum of $\Gamma \backslash \mathbb{H}^{2}$ as the set

$$
\mathcal{L}_{\Gamma}=\left\{k l(\gamma):(k, \gamma) \in \mathbb{N}_{0} \times \mathcal{P}\right\} .
$$

If we view $\Gamma$ as a subgroup of $\mathrm{PSL}_{2}(\mathbb{R})$, then there is a one-to-one correspondence between closed geodesics on $X$ and conjugacy classes of hyperbolic elements in $\Gamma$. If $\gamma$ is a closed geodesic (not necessarily primitive) corresponding to $g_{\gamma} \in \Gamma$, a representative of a conjugacy class, then its length $l(\gamma)$ is related to the trace of $g_{\gamma}$ through the formula

$$
\left|\operatorname{Tr}\left(g_{\gamma}\right)\right|=2 \cosh (l(\gamma) / 2) .
$$

We now recall a few basic facts on the properties of the length spectrum of arithmetic groups. An arithmetic Fuchsian group is defined as a discrete subgroup $\Gamma$ of $\mathrm{PSL}_{2}(\mathbb{R})$ which is commensurable to a subgroup $\Gamma_{0}$ derived from a quaternion algebra, we refer to the book of Katok [15] for precise definitions. Arithmetic Fuchsian groups are widely used in quantum chaos, see for instance the paper [19]. Stating the detailed definition of Fuchsian groups derived from quaternion algebras would be too long and useless here. For our purpose, we will only need the following important property due to Takeuchi [27] which is actually a characterization of arithmetic groups. 
Theorem 3.4 (Takeuchi). Let $\Gamma$ be a discrete, cofinite subgroup of $P S L_{2}(\mathbb{R})$. Set $\operatorname{Tr}(\Gamma):=\{\operatorname{Tr}(T): T \in \Gamma\}, \operatorname{Tr}(\Gamma)^{2}:=\left\{(\operatorname{Tr}(T))^{2}: T \in\right.$ $\Gamma\}$. Then $\Gamma$ is arithmetic if and only if:

(1) The field $K=\mathbb{Q}(\operatorname{Tr}(\Gamma))$ is an algebraic field of finite degree over $\mathbb{Q}$ and $\operatorname{Tr}(\Gamma)$ is a subset of the ring of integers of $K$.

(2) For all embedding $\varphi: K \rightarrow \mathbb{C}$ with $\left.\varphi\right|_{\operatorname{Tr}(\Gamma)^{2}} \neq I d$, the set $\varphi(\operatorname{Tr}(\Gamma))$ is bounded in $\mathbb{C}$.

We recall to the reader that by "embedding" we mean a field morphism $\varphi: K \rightarrow \mathbb{C}$ that leaves $\mathbb{Q}$ invariant. The number of such embeddings is equal to the degree of the extension $K$ over $\mathbb{Q}$. This special features of the trace set $\operatorname{Tr}(\Gamma)$ imply the important bounded cluster property: there exists a constant $C_{\Gamma}>0$ such that for all $n \in \mathbb{N}$,

$$
\# \operatorname{Tr}(\Gamma) \cap[n, n+1] \leq C_{\Gamma} .
$$

A rather easy proof of that fact (based on the number theoretic properties above) can be found in the paper of Luo and Sarnak [19]. The main consequence for our purpose is the following lower bound. Let $\Gamma$ be a convex co-compact subgroup of an arithmetic group $\Gamma_{0}$. For all $\ell \in \mathcal{L}_{\Gamma}$, let $m(\ell)$ denote the multiplicity of $\ell$ as the length of a closed geodesic i.e.

$$
m(\ell):=\#\left\{(k, \gamma) \in \mathbb{N}_{0} \times \mathcal{P}: \ell=k l(\gamma)\right\} .
$$

We have the next result.

Proposition 3.5. Let $\Gamma$ be a convex co-compact subgroup of an arithmetic group. Assume that $\delta(\Gamma)>\frac{1}{2}$, then there exists $A_{\Gamma}>0$ such that for all $T$ large, we have

$$
\sum_{\substack{T-1 \leq \ell \leq T+1 \\ \ell \in \mathcal{L}_{\Gamma}}} m^{2}(\ell) \geq A_{\Gamma} \frac{e^{(2 \delta(\Gamma)-1 / 2) T}}{T^{2}} .
$$

Proof. Because of the bounded cluster property, we have

$$
\#\{t \in \operatorname{Tr}(\Gamma): t \leq x\} \leq C_{\Gamma_{0}}[x],
$$

which using formula (3) gives

$$
\#\left\{\ell \in \mathcal{L}_{\Gamma}: \ell \leq T\right\} \leq 2 C_{\Gamma_{0}} \cosh (T / 2) \leq C^{\prime} e^{T / 2},
$$

for all $T$ large and a well chosen $C^{\prime}$. On the other hand, the prime geodesic theorem (which is due to Lalley [16] for convex co-compact groups) applied to $X=\Gamma \backslash \mathbb{H}^{2}$ states that

$$
\#\left\{(k, \gamma) \in \mathbb{N}_{0} \times \mathcal{P}: k l(\gamma) \leq T\right\}=\frac{e^{\delta T}}{\delta T}(1+o(1)),
$$

which gives

$$
\sum_{\substack{T-1 \leq \ell \leq T+1 \\ \ell \in \mathcal{L}_{\Gamma}}} m(\ell) \geq \widetilde{C} \frac{e^{\delta T}}{T}
$$


for some constant $\widetilde{C}>0$ depending on $\Gamma$. Applying Schwarz inequality gives

$$
\widetilde{C} \frac{e^{\delta T}}{T} \leq \sqrt{C^{\prime}} e^{T / 4}\left(\sum_{\substack{T-1 \leq \ell \leq T+1 \\ \ell \in \mathcal{L}_{\Gamma}}} m^{2}(\ell)\right)^{1 / 2}
$$

and the proof is finished.

3.3. Main proof. We can now proceed to the proof of Theorem 1.2. The idea is to work by contradiction and use the approximate trace formula of Proposition 3.1. We will use the following family of test functions with parameters $\xi \in \mathbb{R}$ and $T \geq 0, T$ large.

$$
\varphi_{\xi, T}(x):=e^{-i \xi x} \varphi(x-T),
$$

where $\varphi \in C_{0}^{\infty}(\mathbb{R})$ with support inside $[-2,2], \varphi \geq 0$ and $\varphi(x)=1$ for all $x \in[-1,1]$. We set for $A \geq \operatorname{Re}(s) \geq 0$,

$$
\psi_{\xi, T}(s):=\widehat{\varphi_{\xi, T}}(i s)=e^{-i \xi T} e^{s T} \widehat{\varphi}(\xi+i s) .
$$

Notice that by integrating by parts, for all integer $N \geq 0$, one can find $C_{N}>0$ such that

$$
\left|\psi_{\xi, T}(s)\right| \leq C_{N} \frac{e^{\operatorname{Re}(s) T}}{(1+|\xi+\operatorname{Im}(s)|)^{N}} .
$$

Assume from now on that there exist only finitely many resonances inside the strip $\{\operatorname{Re}(s)>\rho\}$. Fix $\varepsilon>0$ small enough. We set

$$
\mathcal{S}_{\xi, T}:=\sum_{k \in \mathbb{N}_{0}} \sum_{\gamma \in \mathcal{P}} \frac{l(\gamma)}{1-e^{-k l(\gamma)}} e^{-i \xi k l(\gamma)} \varphi(k l(\gamma)-T) .
$$

Applying the approximate trace formula to $\varphi_{\xi, T}$ we have

$$
\mathcal{S}_{\xi, T}=\sum_{\Gamma \in \mathcal{F}_{\rho}} \psi_{\xi, T}(\Gamma)+E(\xi, T),
$$

where the error term $E(\xi, T)$ satisfies

$$
E(\xi, T)=O\left(\int_{-\infty}^{+\infty}(1+|x|)^{\delta}\left|\psi_{\xi, T}(\rho+\varepsilon+i x)\right| d x\right) .
$$

Using the estimate (4) with $N=2$ and the fact that $\delta<1$, we can bound this error term by

$$
E(\xi, T)=O\left(e^{(\rho+\varepsilon) T} I(\xi)\right),
$$

where $I(\xi)$ is the following convergent integral

$$
I(\xi)=\int_{-\infty}^{+\infty} \frac{(1+|x|)^{\delta}}{(1+|\xi+x|)^{2}} d x
$$


An obvious change of variable shows that for $|\xi| \geq 1, I(\xi)$ is bounded from above by

$$
I(\xi)=\int_{-\infty}^{+\infty} \frac{(1+|u-\xi|)^{\delta}}{(1+|u|)^{2}} d u \leq|\xi|^{\delta} \int_{-\infty}^{+\infty} \frac{(2+|u|)^{\delta}}{(1+|u|)^{2}} d u
$$

so that we have

$$
E(\xi, T)=O\left(e^{(\rho+\varepsilon) T}|\xi|^{\delta}\right) .
$$

The key step is now to consider the weighted squared sum ${ }^{2}$

$$
\mathcal{G}(\sigma, T):=\sqrt{\sigma} \int_{-\infty}^{+\infty} e^{-\sigma \xi^{2}}\left|\mathcal{S}_{\xi, T}\right|^{2} d \xi
$$

where $\sigma>0$ is a small parameter depending on $T$ (which is large) to be adjusted later on. We also set for $(k, \gamma),\left(k^{\prime}, \gamma^{\prime}\right) \in \mathbb{N}_{0} \times \mathcal{P}$,

$$
a_{(k, \gamma) ;\left(k, \gamma^{\prime}\right)}:=\frac{l(\gamma) l\left(\gamma^{\prime}\right)}{\left(1-e^{-k l(\gamma)}\right)\left(1-e^{-k^{\prime} l\left(\gamma^{\prime}\right)}\right)} .
$$

Interchanging summations we have

$$
\begin{gathered}
\mathcal{G}(\sigma, T)=\sum_{(k, \gamma),\left(k^{\prime}, \gamma^{\prime}\right) \in \mathbb{N}_{0} \times \mathcal{P}} a_{(k, \gamma) ;\left(k^{\prime}, \gamma^{\prime}\right)} \varphi(k l(\gamma)-T) \varphi\left(k^{\prime} l\left(\gamma^{\prime}\right)-T\right) \\
\times \int_{-\infty}^{+\infty} e^{-i \xi\left(k l(\gamma)-k^{\prime} l\left(\gamma^{\prime}\right)\right)} e^{-\sigma \xi^{2}} \sqrt{\sigma} d \xi .
\end{gathered}
$$

Using the classical formula for the Fourier transform of Gaussian functions, we obtain

$$
\begin{gathered}
\mathcal{G}(\sigma, T)=\sqrt{\pi} \sum_{(k, \gamma),\left(k^{\prime}, \gamma^{\prime}\right) \in \mathbb{N}_{0} \times \mathcal{P}} a_{(k, \gamma) ;\left(k^{\prime}, \gamma^{\prime}\right)} \varphi(k l(\gamma)-T) \varphi\left(k^{\prime} l\left(\gamma^{\prime}\right)-T\right) \\
\times e^{-\frac{\left(k l(\gamma)-k^{\prime} l\left(\gamma^{\prime}\right)\right)^{2}}{4 \sigma}} .
\end{gathered}
$$

Since all summands in the above formula are positive, we can simply drop all terms with $k l(\gamma) \neq k^{\prime} l\left(\gamma^{\prime}\right)$ to get the lower bound

$$
\mathcal{G}(\sigma, T) \geq C \sum_{\substack{T-1 \leq \ell \leq T+1 \\ \ell \in \mathcal{L}_{\Gamma}}} m^{2}(\ell),
$$

for all $T$ large and a suitable constant $C>0$. We recall that $\mathcal{L}_{\Gamma}$ is the length spectrum and $m(\ell)$ is the multiplicity of a given length.

\footnotetext{
${ }^{2}$ There is nothing sacred about the gaussian weight here. One can use any "squared convoluted" weight $W_{\sigma}(\xi)$ of the form

$$
W_{\sigma}(\xi)=f \star f(\sqrt{\sigma} \xi),
$$

where $f \geq 0$ is in Schwartz class so that the Fourier transform of $W_{\sigma}(\xi)$ is positive.
} 
Going back to (5), we have

$$
\frac{\mathcal{G}(\sigma, T)}{2 \sqrt{\sigma}} \leq \underbrace{\int_{-\infty}^{+\infty} e^{-\sigma \xi^{2}}\left|\sum_{\Gamma \in \mathcal{F}_{\rho}} \psi_{\xi, T}(\Gamma)\right|^{2} d \xi}_{\mathcal{J}_{1}(\sigma, T)}+\underbrace{\int_{-\infty}^{+\infty} e^{-\sigma \xi^{2}}|E(\xi, T)|^{2} d \xi}_{\mathrm{J}_{2}(\sigma, T)} .
$$

Using the estimate (6), one obtains that

$$
\begin{aligned}
\mathcal{J}_{2}(\sigma, T) & =O\left(e^{2(\rho+\varepsilon) T} \int_{-\infty}^{+\infty} e^{-\sigma \xi^{2}}|\xi|^{2 \delta} d \xi\right) \\
& =O\left(e^{2(\rho+\varepsilon) T} \sigma^{-\delta-\frac{1}{2}}\right) .
\end{aligned}
$$

On the other hand, since $\mathcal{F}_{\rho}$ is finite and using estimate (4) with $N=1$, one can find $\widetilde{C}>0$ such that for all $\Gamma \in \mathcal{F}_{\rho}$,

$$
\left|\psi_{\xi, T}(\Gamma)\right| \leq \widetilde{C} \frac{e^{\delta T}}{(1+|\xi|)},
$$

which shows that

$$
\mathcal{J}_{1}(\sigma, T)=O\left(e^{2 \delta T} \int_{-\infty}^{+\infty} \frac{e^{-\sigma \xi^{2}}}{(1+|\xi|)^{2}} d \xi\right)=O\left(e^{2 \delta T}\right),
$$

uniformly in $\sigma$. We can now conclude the proof, depending on $\delta$.

In general, we have no effective lower bound on the multiplicity $m(\ell)$ and have to rely on the lower bound

$$
\mathcal{G}(\sigma, T) \geq C \sum_{\substack{T-1 \leq \ell \leq T+1 \\ \ell \in \mathcal{L}_{\Gamma}}} m(\ell)
$$

which by the prime orbit theorem yields

$$
\mathcal{G}(\sigma, T) \geq B \frac{e^{\delta T}}{T}
$$

for some well chosen $B>0$. We have therefore obtained for all $T$ large and $\sigma$ small,

$$
B \frac{e^{\delta T}}{T}=O\left(\sqrt{\sigma} e^{2 \delta T}\right)+O\left(e^{2(\rho+\varepsilon) T} \sigma^{-\delta}\right) .
$$

Setting $\sigma=e^{-\alpha T}$, we have a contradiction as $T \rightarrow+\infty$ provided

$$
\alpha>2 \delta \text { and } \rho<\frac{\delta(1-\alpha)}{2}-\varepsilon,
$$

which shows ( $\varepsilon$ being as small as one wants) that there are infinitely many resonances in the strip $\left\{\operatorname{Re}(s) \geq \frac{\delta(1-2 \delta)}{2}-\epsilon\right\}$ for all $\epsilon>0$. 
If $\delta>\frac{1}{2}$ and $\Gamma$ is a subgroup of an arithmetic group, we can use the lower bound of Proposition 3.5 to take advantage of the large multiplicities in the length spectrum and write (for some $B>0$ )

$$
B \frac{e^{\left(2 \delta-\frac{1}{2}\right) T}}{T^{2}}=O\left(\sqrt{\sigma} e^{2 \delta T}\right)+O\left(e^{2(\rho+\varepsilon) T} \sigma^{-\delta}\right),
$$

which if $\sigma=e^{-\alpha T}$ produces a contradiction whenever $\alpha>1$ and

$$
\rho<\frac{\delta(2-\alpha)}{2}-\frac{1}{4}-\varepsilon
$$

As above, we have found infinitely many resonances (or zeros of $Z_{\Gamma}(s)$ ) in the strip $\left\{\operatorname{Re}(s) \geq \frac{\delta}{2}-\frac{1}{4}-\epsilon\right\}$ for all $\epsilon>0$.

\section{REFERENCES}

[1] David Borthwick. Spectral theory of infinite-area hyperbolic surfaces, volume 256 of Progress in Mathematics. Birkhäuser Boston Inc., Boston, MA, 2007.

[2] David Borthwick, Chris Judge, and Peter A. Perry. Selberg's zeta function and the spectral geometry of geometrically finite hyperbolic surfaces. Comment. Math. Helv., 80(3):483-515, 2005.

[3] Jean Bourgain and Alex Kontorovich. On representations of integers in thin subgroups of $\mathrm{SL}_{2}(\mathbf{Z})$. Geom. Funct. Anal., 20(5):1144-1174, 2010.

[4] Jean Bourgain, Alex Gamburd, and Peter Sarnak. Generalization of Selberg's 3/16 Theorem and Affine sieve. Arxiv preprint, 2009.

[5] Lewis Bowen. Free groups in lattices. Geom. Topol., 13(5):3021-3054, 2009.

[6] Rufus Bowen. Hausdorff dimension of quasicircles. Inst. Hautes Études Sci. Publ. Math., (50):11-25, 1979.

[7] Tania Christiansen and Maciej Zworski. Resonance wave expansions: two hyperbolic examples. Comm. Math. Phys., 212(2):323-336, 2000.

[8] Alex Gamburd. On the spectral gap for infinite index "congruence" subgroups of $\mathrm{SL}_{2}(\mathbf{Z})$. Israel J. Math., 127:157-200, 2002.

[9] Colin Guillarmou and Frédéric Naud. Wave 0-trace and length spectrum on convex co-compact hyperbolic manifolds. Comm. Anal. Geom., 14(5):945-967, 2006.

[10] Colin Guillarmou and Frédéric Naud. Wave decay on convex co-compact hyperbolic manifolds. Comm. Math. Phys., 287(2):489-511, 2009.

[11] Laurent Guillopé and Maciej Zworski. The wave trace for Riemann surfaces. Geom. Funct. Anal., 9(6):1156-1168, 1999.

[12] Laurent Guillopé, Kevin Lin, and Maciej Zworski. The Selberg zeta function for convex co-compact Schottky groups. Comm. Math. Phys., 245(1):149-176, 2004.

[13] Laurent Guillopé and Maciej Zworski. Upper bounds on the number of resonances for non-compact Riemann surfaces. J. Funct. Anal., 129(2):364-389, 1995.

[14] Laurent Guillopé and Maciej Zworski. Scattering asymptotics for Riemann surfaces. Ann. of Math. (2), 145(3):597-660, 1997.

[15] Svetlana Katok. Fuchsian groups. Chicago Lectures in Mathematics. University of Chicago Press, Chicago, IL, 1992.

[16] Steven P. Lalley. Renewal theorems in symbolic dynamics, with applications to geodesic flows, non-Euclidean tessellations and their fractal limits. Acta Math., 163(1-2):1-55, 1989. 
[17] Peter D. Lax and Ralph S. Phillips. The asymptotic distribution of lattice points in Euclidean and non-Euclidean spaces. J. Funct. Anal., 46:280-350, 1982.

[18] Peter D. Lax and Ralph S. Phillips. Translation representation for automorphic solutions of the non-Euclidean wave equation I, II, III. Comm. Pure. Appl. Math., 37,38:303-328, 779-813, 179-208, 1984, 1985.

[19] Wenzhi Luo and Peter Sarnak. Number variance for arithmetic hyperbolic surfaces. Comm. Math. Phys., 161(2):419-432, 1994.

[20] Rafe R. Mazzeo and Richard B. Melrose. Meromorphic extension of the resolvent on complete spaces with asymptotically constant negative curvature. $J$. Funct. Anal., 75(2):260-310, 1987.

[21] Frédéric Naud. Expanding maps on Cantor sets and analytic continuation of zeta functions. Ann. Sci. École Norm. Sup. (4), 38(1):116-153, 2005.

[22] Frédéric Naud. Heat kernels and the rate of mixing of compact extensions of expanding maps. Preprint, 2010.

[23] Samuel J. Patterson. The limit set of a Fuchsian group. Acta Math., 136(34):241-273, 1976.

[24] Samuel J. Patterson and Peter A. Perry. The divisor of Selberg's zeta function for Kleinian groups. Duke Math. J., 106(2):321-390, 2001. Appendix A by Charles Epstein.

[25] David Ruelle. Zeta-functions for expanding maps and Anosov flows. Invent. Math., 34(3):231-242, 1976.

[26] Atle Selberg. Remarks on the distribution of poles of Eisenstein series. In Festschrift in honor of I. I. Piatetski-Shapiro on the occasion of his sixtieth birthday, Part II (Ramat Aviv, 1989), volume 3 of Israel Math. Conf. Proc., pages 251-278. Weizmann, Jerusalem, 1990.

[27] Kisao Takeuchi. A characterization of arithmetic Fuchsian groups. J. Math. Soc. Japan, 27(4):600-612, 1975.

[28] Edward C. Titchmarsh. The theory of functions. Oxford University Press, second edition, 1932.

McGill university, Department of Mathematics and Statistics, MonTreal, Quebec, Canada H3A2K6.

E-mail address: jakobson@math.mcgill.ca

Laboratoire D'Analyse non-Linéaire et GÉométrie (EA 2151), Université d'Avignon et des pays de Vaucluse, F-84018 Avignon, France.

E-mail address: frederic.naud@univ-avignon.fr 